1 Universidade Federal de Pernambuco (UFPE) Recife (PE), Brasil. isabelle_rayanne@hotmail. com

2 Universidade Federal de Pernambuco (UFPE), Departamento de Medicina Social - Recife (PE), Brasil.

marciacarrera@hotmail.com

3 Universidade Federal de Pernambuco (UFPE), Departamento de Medicina Social - Recife (PE), Brasil.

marquesap@hotmail.com

4 Universidade do Porto (UP), Instituto de Ciências Biomédicas Abel Salazar Porto, Portugal.

july_nurse21@hotmail.com

\section{Fatores associados à depressão em idosos institucionalizados: revisão integrativa}

\author{
Factors associated with depression in institutionalized elders: \\ integrative review
}

Isabelle Rayanne Alves Pimentel da Nóbrega1, Márcia Carréra Campos Leal2, Ana Paula de

Oliveira Marques³, Júlia de Cássia Miguel Vieira4

RESUMO A presente revisão integrativa da literatura objetivou verificar o conhecimento científico produzido relacionado aos fatores significativamente associados à sintomatologia depressiva em idosos residentes em Instituições de Longa Permanência. Foram consultadas as bases de dados Lilacs, Medline e Ibecs, entre 2002 e 2012, cuja amostra de vinte artigos identificou seis grupos de fatores associados à depressão em idosos institucionalizados: sociodemográficos, condições de saúde, capacidade funcional, comportamento, cognição e medicamentos. Espera-se que os resultados desta revisão contribuam para a reflexão sobre as práticas de saúde destinadas aos idosos que vivem no âmbito institucional.

PALAVRAS-CHAVE Idoso; Depressão; Instituição de longa permanência para idosos.

ABSTRACT This integrative literature review aimed to assess the scientific knowledge related to factors significantly associated with depressive symptoms in elderly residents in long-stay institutions. The databases Lilacs, Medline and Ibecs were consulted from 2002 to 2012, which provided a twenty articles sample that allowed us to identify six main groups of factors associated with depression among institutionalized elders: sociodemographic, health status, functional ability, behavior, cognition and drugs. It is expected that the results of this review may contribute to the debate on health practices for older people living in institutional ambience.

KEYWORDS Aged; Depression; Homes for the aged. 


\section{Introdução}

O envelhecimento populacional é um fenômeno de abrangência mundial, sendo atualmente mais expressivo e impactante nos países em desenvolvimento (BATISTA ET AL., 2008). No Brasil, o crescimento abrupto da população idosa resulta da combinação de variáveis estritamente demográficas com as profundas alterações sociais e culturais ocorridas, que simultaneamente configuram-se como causa e consequência (CRUZ; CAETANO; LEITE, 2010).

Em 2011, dos estimados 195,2 milhões de habitantes do País, 12,1\% eram de pessoas com 60 anos ou mais de idade (IBGE, 2012). Projeções apontam que em 2050 esse contingente atingirá 38 milhões de idosos, superando a proporção de jovens na população (BRASIL, 2012).

As mudanças no perfil etário brasileiro, entretanto, não têm sido devidamente acompanhadas de reorganização das políticas públicas, estando ainda o setor da saúde despreparado para atender à demanda de uma população cada vez mais envelhecida e com uma sobrecarga de doenças crônico-degenerativas que levam a limitações funcionais e cognitivas (SAMPAIO ET AL., 2009; VALCARENGHI, 2011).

A instabilidade econômica e o agravamento das condições de saúde geralmente trazem o idoso para mais perto de seus familiares, que nem sempre aceitam ou estão aptos à função de cuidadores, aumentando a demanda por Instituições de Longa Permanência para Idosos (Ilpi) (GALHARDO; MARIOSA; TAKATA, 2010). Essas instituições, governamentais ou não, possuem caráter residencial e são destinadas ao domicílio coletivo de pessoas idosas, com ou sem suporte familiar, em condições de liberdade, dignidade e cidadania, oferecendo-lhes alimentação, moradia e lazer (BRASIL, 2005).

No entanto, em muitas delas, a falta de estrutura física adequada e recursos humanos capacitados, aliada à assistência caritativa e protecionista, frequentemente insensíveis às potencialidades do idoso e à sua liberdade de escolha, pode aumentar o quadro de dependência, o isolamento social e a falta de perspectivas para uma vida ativa e com qualidade (SOUZA ET AL., 2011).

O contexto institucional também favorece ao idoso vivenciar perdas em vários aspectos da vida, aumentando a vulnerabilidade a quadros depressivos que podem desencadear desordens psiquiátricas, perda da autonomia e agravamento de quadros patológicos preexistentes (CARREIRA ET AL., 2011).

A depressão caracteriza-se como um distúrbio de natureza multifatorial da área afetiva ou do humor, que exerce forte impacto funcional e envolve inúmeros aspectos de ordem biológica, psicológica e social, tendo como principais sintomas o humor deprimido e a perda de interesse ou prazer em quase todas as atividades (CARREIRA ET AL., 2011).

Atualmente, é apontada como o quarto maior agente incapacitante das funções sociais e de outras atividades da vida cotidiana, sendo responsável por cerca de 850 mil mortes a cada ano (GIAVONI ET AL., 2008). Também é considerada a enfermidade mental de maior prevalência em nível mundial e estima-se que em 2020 será a segunda causa global de incapacidade (GONZÁLeZ ET AL., 2010).

Na população idosa, essa é uma doença comum, recorrente e frequentemente subdiagnosticada e subtratada, principalmente em nível de cuidados de saúde primários (MEDEIROS, 2010). Epidemiologicamente, estima-se que aproximadamente $15 \%$ dos idosos apresentam sintomas de depressão, sendo essa prevalência maior nas populações institucionalizadas (SANTANA; BARBOZA FILHO, 2007; PÓVOA ET AL., 2009; SIQUEIRA ET AL., 2009).

Nessa fase da vida, a sintomatologia depressiva é permeada por elementos que dizem respeito não apenas à doença, mas às oscilações sentimentais próprias do envelhecimento e ao contexto social marcado pelo culto aos valores da juventude (HARTMANN JUNIOR; SILVA; BASTOS, 2009). É também no indivíduo idoso que a depressão tem pior 
prognóstico e maior incidência de suicídios, podendo, quando duradouros, interferir na sua capacidade funcional, de autocuidado e nas suas relações sociais (FERNANDES; NASCIMENTO; COSTA, 2010).

Vários autores têm estudado os estados depressivos nos idosos. No entanto, a maioria dos artigos enfoca os fatores biológicos da doença, com pouco destaque para os aspectos psicossociais associados ao transtorno (HARTMANN JUNIOR; SILVA; BASTOS, 2009).

Frente a essa problemática e à relevância do tema exposto, justifica-se a necessidade de buscar evidências que apontem quais os fatores associados à depressão em pessoas idosas que vivem no ambiente institucional, no intuito de condensar e disseminar o conhecimento produzido a respeito e auxiliar na melhoria da atenção ofertada a esses indivíduos.

O presente estudo teve como objetivo verificar o conhecimento científico produzido relacionado aos fatores significativamente associados à sintomatologia depressiva em pessoas idosas residentes em Ilpi.

\section{Procedimentos metodológicos}

Dentre os métodos de revisão de literatura, o presente estudo adotou a modalidade integrativa, que é considerada a mais ampla abordagem metodológica referente às revisões por permitir a inclusão de estudos experimentais e não experimentais, combinando dados teóricos e empíricos, no intuito de fornecer uma vasta compreensão sobre o fenômeno analisado (SOUZA; SILVA; CARVALHO, 2010).

A revisão integrativa da literatura é comumente empregada na análise de conceitos, revisão de teorias ou evidências e síntese do conhecimento sobre determinado assunto, permitindo identificar lacunas que precisam ser preenchidas com a realização de novos estudos, sendo também bastante plausível na busca de evidências para a prática clínica dos profissionais da área de saúde (OLIVEIRA, 2008; FARIA, 2010).

Para o desenvolvimento desta revisão, foram percorridas as seguintes etapas: 1) definição do tema e formulação da questão norteadora, 2) escolha das bases de dados eletrônicos utilizadas na pesquisa, 3) estabelecimento dos critérios de inclusão e exclusão, 4) definição dos descritores, 5) pré-seleção dos artigos, 6) avaliação dos estudos pré-selecionados e seleção dos estudos incluídos na revisão, 7) interpretação dos resultados e 8) apresentação da revisão integrativa.

A coleta de dados ocorreu durante o mês de janeiro de 2013 e foi norteada pela seguinte pergunta: 'Quais as evidências científicas que retratam os fatores significativamente associados à depressão em pessoas idosas institucionalizadas?' Foram empregados os Descritores em Ciências da Saúde (DeCS): idoso, depressão e instituição de longa permanência para idosos. Os critérios de inclusão adotados foram:

- ser artigo e ter sido publicado entre os anos de 2002 e 2012 nas bases de dados Literatura Latino-Americana em Ciências da Saúde (Lilacs), Medical Literature and Retrieval System Online (Medline) e Índice Bibliográfico Español em Ciencias de la Salud (Ibecs);

- ter sido divulgado em língua portuguesa, inglesa ou espanhola;

- estar disponível na íntegra;

- apresentar em seus resultados fatores significativamente associados à sintomatologia depressiva em pessoas idosas institucionalizadas.

Seguindo critérios de exclusão, foram desconsideradas publicações referentes a teses, dissertações, resumos de congressos, anais, editoriais, comentários e opiniões, artigos de revisão, estudos que incluíssem 
em sua amostra pessoas com idade inferior a 60 anos e que encontrassem associação estatística não significativa entre depressão e os fatores estudados.

A estratégia de busca utilizada combinou dois ou mais descritores por meio do conector 'and' no campo 'descritor de assunto'. A partir daí, foi realizada a pré-seleção dos artigos relacionados ao tema por meio da leitura criteriosa dos títulos e resumos, a fim de verificar a adequação dos estudos aos critérios de inclusão.

Os artigos pré-selecionados foram lidos na íntegra e aqueles selecionados para a revisão foram analisados a partir de dois instrumentos. O primeiro, adaptado do Critical Apppraisal Skills Programme (Casp) Programa de habilidades em leitura crítica, integrante do Public Health Resource Unit (PHRU), elaborado pela Universidade de Oxford, em 1993 (LONDON, 2002). O instrumento foi eleito devido à proposta de análise objetiva, sistemática e de fácil entendimento. Este consiste em 10 itens (máximo 10 pontos), abrangendo: 1) objetivo, 2) adequação metodológica, 3) apresentação dos procedimentos teóricos e metodológicos, 4) seleção da amostra, 5) procedimento para a coleta de dados, 6) relação entre o pesquisador e pesquisados, 7) consideração dos aspectos éticos, 8) procedimento para a análise dos dados, 9) apresentação dos resultados e 10) importância da pesquisa. Os estudos foram classificados de acordo com as seguintes pontuações: 6 a 10 pontos - boa qualidade metodológica e viés reduzido; e mínima de 5 pontos qualidade metodológica satisfatória, porém com risco de viés aumentado. Neste estudo, optou-se por utilizar apenas os artigos classificados de 6 a 10 pontos.

$\mathrm{O}$ segundo instrumento correspondeu à Classificação Hierárquica das Evidências para Avaliação de Estudos, baseado na categorização da Agency for Healthcare Research and Quality (AHRQ), onde a qualidade das evidências é classificada em sete níveis: I) revisão sistemática ou metanálise, II) ensaios clínicos randomizados, III) ensaio clínico sem randomização, IV) estudos de coorte e de caso-controle, V) revisão sistemática de estudos descritivos e qualitativos, VI) único estudo descritivo ou qualitativo e VII) opinião de autoridades e/ou relatório de comitês de especialidades (MELNYK ET AL., 2010). Atendendo aos critérios de exclusão, foram considerados apenas os estudos com níveis II, III, IV e VI de evidência.

A análise dos artigos ocorreu de forma descritiva, de acordo com o instrumento de coleta de dados, permitindo verificar as seguintes características de cada pesquisa: autoria, periódico, país de origem, idioma, delineamento da pesquisa, ano da publicação, instrumento utilizado e fatores associados à depressão.

\section{Resultados e discussão}

Na presente revisão integrativa, foram analisados vinte artigos que atenderam aos critérios de inclusão previamente estabelecidos. A tabela 1 apresenta os resultados das buscas pelos descritores de acordo com as bases de dados.

Tabela 1. Distribuição dos artigos encontrados e selecionados por base de dados. Recife (PE), 2013

\begin{tabular}{lrrrr}
\hline Base de dados & Encontrados & Pré-secionados & Excluídos & Analisados \\
\hline Ibecs & 13 & 0 & - & - \\
Lilacs & 786 & 5 & 4 & 1 \\
Medline & 27069 & 28 & 9 & 19 \\
\hline Total & 27868 & 33 & 13 & 20 \\
\hline
\end{tabular}

Fonte: Elaboração própria 
Dentre os artigos analisados, quatro apresentam médicos como autores, um é de autoria de dois enfermeiros e uma estatística e nos demais não foi possível identificar a categoria profissional dos autores.

Em relação aos tipos de periódicos nos quais foram publicados os artigos incluídos na revisão, onze faziam parte de revistas de psiquiatria geriátrica, dois de revistas de psiquiatria geral, três foram publicados em revistas sobre envelhecimento, geriatria e gerontologia, dois em revistas de nutrição, um em revista médica e um em revista de enfermagem clínica.

Quanto ao país de origem das pesquisas, oito artigos são provenientes de países europeus, cinco de países asiáticos, três são originários da América do Norte (Estados Unidos), três da América do Sul (sendo dois destes do Brasil) e um foi realizado na Oceania (Austrália).

Apenas um artigo, aquele publicado na base Lilacs, estava no idioma espanhol, sendo as demais publicações de língua inglesa. Em relação às instituições de origem das pesquisas, dos vinte artigos, somente um estava vinculado a um centro de estudos, enquanto os demais foram promovidos por universidades e/ou hospitais.

No que se refere ao delineamento da pesquisa, apenas um estudo era do tipo coorte prospectiva (nível IV de evidência) enquanto os demais eram descritivos transversais (nível VI de evidência). Dentre os estudos transversais, quatro baseavam-se na análise de dados secundários, provenientes de pesquisas prévias. Segundo Toledo (2008), a análise do desenho é importante, visto que aponta a ótica em que o problema foi analisado.

Dentro do intervalo cronológico considerado na revisão, apenas os anos de 2002 e 2011 não apresentaram estudos compatíveis com os critérios de inclusão, o que demonstra a constante atualização desta temática.

O quadro 1 apresenta uma síntese da caracterização dos artigos segundo título, autores, periódico onde foi publicado, país de origem do estudo, delineamento da pesquisa e ano da publicação.

Quadro 1. Distribuição dos artigos incluídos na revisão integrativa segundo título, autor, periódico, país de origem, delineamento da pesquisa e ano da publicação

\begin{tabular}{|c|c|c|c|c|c|c|}
\hline № & Título & Autores & Periódico & País & $\begin{array}{c}\text { Delineamento da } \\
\text { pesquisa }\end{array}$ & Ano \\
\hline 01 & $\begin{array}{l}\text { Association of Depression with } \\
\text { Agitation in Elderly Nursing Home } \\
\text { Residents }\end{array}$ & $\begin{array}{l}\text { HEEREN, O.; BORIN, L.; } \\
\text { RASKIN, A.; GRUBER- } \\
\text { BALDINI, A. L.; MENON, A. } \\
\text { S.; KAUP, B.; LORECK, D.; } \\
\text { RUSKIN, P. E.; ZIMMERMAN, } \\
\text { S.; MAGAZINER, J. }\end{array}$ & $\begin{array}{l}\text { Journal of Geriatric } \\
\text { Psychiatry and } \\
\text { Neurology }\end{array}$ & $\begin{array}{l}\text { Estados } \\
\text { Unidos }\end{array}$ & $\begin{array}{l}\text { Estudo transversal } \\
\text { quantitativo - } \\
\text { análise de dados } \\
\text { secundários }\end{array}$ & 2003 \\
\hline 02 & $\begin{array}{l}\text { Risk indicators of depression in } \\
\text { residential homes }\end{array}$ & $\begin{array}{l}\text { EISSES, A. M. H.; KLUITER H.; } \\
\text { JONGENELIS, K.; POT A. M.; } \\
\text { BEEKMAN, A. T. F.; ORMEL, J. }\end{array}$ & $\begin{array}{l}\text { International Journal of } \\
\text { Geriatric Psychiatry }\end{array}$ & Holanda & $\begin{array}{l}\text { Estudo transversal } \\
\text { quantitativo - } \\
\text { análise de dados } \\
\text { secundários }\end{array}$ & 2004 \\
\hline 03 & $\begin{array}{l}\text { The prevalence of depressive } \\
\text { symptoms among elderly } \\
\text { Chinese private nursing home } \\
\text { residents in Hong Kong }\end{array}$ & $\begin{array}{l}\text { CHOW, E. S. L.; KONG, B. M; } \\
\text { H.; WONG, M. T. P.; DRAPER, } \\
\text { B.; LIN K. L.; HO, S. K. S.; } \\
\text { WONG, C. P. }\end{array}$ & $\begin{array}{l}\text { International Journal of } \\
\text { Geriatric Psychiatry }\end{array}$ & China & $\begin{array}{l}\text { Estudo transversal } \\
\text { quantitativo }\end{array}$ & 2004 \\
\hline
\end{tabular}


Quadro 1. (cont.)

04 Comparison of the prevalence and risk factors for depressive symptoms among elderly nursing home residents in

Taiwan and Hong Kong

05 Depression as prognostic factor: 6 months follow-up in a geriatric institution

06 Depressive symptoms and cognitive performance of the elderly: relationship between institutionalization and activity programs

07 Prevalence and risk factors for depressive symptoms among veterans home elders in Eastern Taiwan

08 Incidence and Outcome of Depressive Symptoms in Nursing Home Patients in The Netherlands

09 Assisted Living Policies Promoting Autonomy and Their Relationship to Resident Depressive Symptoms

10 Recognising depression in residential facilities: an Australian challenge

11 Prevalence and symptomatology of depression in older people living in institutions in England and Wales

12 Relación entre los procesos cognitivos y el nivel de depresión en las adultas mayores institucionalizadas en el Asilo San Antonio de Bucaramanga

13 Functional status and depressive symptoms among older adults from residential care facilities in the Czech Republic

14 Relationships between health status, depression and cognitive functions of institutionalized male veterans

15 Dependence of the Geriatric Depression scores on age, nutritional status, and haematologic variables in elderly institutionalized patients

16 Malnutrition and depression in the institutionalised elderly

17 Depression in Dutch homes for the elderly: under-diagnosis in demented residents?
TSAI, Y.; CHUNG, J. W. Y.; WONG, T. K. S.; HUANG, C. M

International Journal of China

Estudo transversal

2005

SHIM, Y. S, YANG, D.

PLATI, M. C. F.; COVRE, P.; LUKASOVA, K.; MACEDO, E. C.

KU, Y.; LIU, W.; TSAI, Y

SMALBRUGGE, M.; JONGENELIS, M.POT, A. M. EEFSTING, J. A.; RIBBE, M. W.; BEEKMAN, A. T. F.

CHEN, C. K.: ZIMMERMAN S.; SLOANE, P. D.; BARRICK, A. L.

SNOWDON, J.; FLEMING, R.

MCDOUGALL, F. A.; MATTHEWS, F. E.; KVAAL, K.; DEWEY, M. E.; BRAYNE, C.

CÓRDOBA, A. M. C.

$\begin{array}{ll}\text { VANKOVA, H.; HOLMEROVA, } & \text { International Journal of } \\ \text { I.; ANDEL, R.; VELETA, P.; } & \text { Geriatric Psychiatry }\end{array}$

JANECKOVA, $\mathrm{H}$.

CHUNG, P.; CHIOU, C.;

$\mathrm{CHOU}, \mathrm{F}$.

ALVES DE REZENDE, C. H.; COELHO, L. M.; OLIVEIRA, L. Nutrition, Health \& M.; PENHA-SILVA, N.

SMOLINER, C.; NORMAN, K.; British Journal of WAGNER, K.; HARTIG, W.; Nutrition LOCHS, H.; PIRLICH, M.

BALLER, M.; BOORSMA M.; FRIJTERS, D. H. M.; MARWIJK, H. W. J.; NIJPELS, G.; HOUT, H. P. J. Geriatric Psychiatry

Universitas

Psychologica

Gerontology and

Geriatrics

Revista Brasileira de Brasil

Psiquiatria

International Journal of China

Geriatric Psychiatry

American Journal of

Holanda

American Journal of Geriatric Psychiatry

International Journal of Geriatric Psychiatry

Age and Ageing

República

Archives of

Gerontology and

Geriatrics

The Journal of Aging

quantitativo

Coréia do Estudo de coorte

2006

rospectivo

Estudo transversal

2006

quantitativo

Estudo transversal

2006

quantitativo

Estudo transversal

2006

quantitativo -

análise de dados

secundários

Estados Estudo transversal

2007

Unidos

quantitativo -

análise de dados secundários

2007 quantitativo

Estudo transversa

2007

Unido quantitativo

Tcheca

Estudo transversa

2008 quantitativo

Estudo transversa

2009 quantitativo

Brasil

Estudo transversal

2009 quantitativo

Alemanha

Estudo transversa

2009 quantitativo

International Journal of Geriatric Psychiatry
Estudo transversa quantitativo
Estudo transversa 


\begin{tabular}{|c|c|c|c|c|c|c|}
\hline \multicolumn{7}{|c|}{ Quadro 1. (cont.) } \\
\hline 18 & $\begin{array}{l}\text { Depression in the first year of stay } \\
\text { for elderly long-term nursing home } \\
\text { residents in the U.S.A. }\end{array}$ & $\begin{array}{l}\text { HOOVER, D. R.; SIEGEL, } \\
\text { M.; LUCAS, J.; KALAY, E.; } \\
\text { GABODA, D.; DEVANAND, D. } \\
\text { P.; CRYSTAL, S. }\end{array}$ & $\begin{array}{l}\text { International } \\
\text { Psychogeriatrics }\end{array}$ & $\begin{array}{l}\text { Estados } \\
\text { Unidos }\end{array}$ & $\begin{array}{l}\text { Estudo transversal } \\
\text { quantitativo }\end{array}$ & 2010 \\
\hline 19 & $\begin{array}{l}\text { Descriptive Epidemiology of } \\
\text { Undetected Depression in } \\
\text { Institutionalized Older People }\end{array}$ & $\begin{array}{l}\text { DAMIÁN, J.; PASTOR- } \\
\text { BARRIUSO, R.; } \\
\text { VALDERRAMA-GAMA, E. }\end{array}$ & $\begin{array}{l}\text { Journal of the } \\
\text { American Medical } \\
\text { Directors Association }\end{array}$ & Espanha & $\begin{array}{l}\text { Estudo transversal } \\
\text { quantitativo }\end{array}$ & 2010 \\
\hline 20 & $\begin{array}{l}\text { The impact of depression and sense } \\
\text { of coherence on emotional and } \\
\text { social loneliness among nursing } \\
\text { home residents without cognitive } \\
\text { impairment - a questionnaire survey }\end{array}$ & $\begin{array}{l}\text { DRAGESET, J.; ESPEHAUG, B.; } \\
\text { KIRKEVOLD, M. }\end{array}$ & $\begin{array}{l}\text { Journal of Clinical } \\
\text { Nursing }\end{array}$ & Noruega & $\begin{array}{l}\text { Estudo transversal } \\
\text { quantitativo }\end{array}$ & 2012 \\
\hline
\end{tabular}

Fonte: Elaboração própria

A partir da leitura minuciosa dos artigos
analisados, foi possível identificar quais
fatores encontravam-se significativamente as-
sociados aos sintomas depressivos nos idosos
vivendo em condição de institucionalização.

O quadro 2 apresenta, para cada estudo incluído na revisão, os instrumentos utilizados na detecção de sintomatologia depressiva, bem como os resultados referentes aos testes de associação feitos para os fatores investigados.

Quadro 2. Distribuição dos artigos incluídos na revisão integrativa segundo instrumento utilizado na coleta de dados e resultados referentes aos fatores associados à depressão em idosos institucionalizados

\begin{tabular}{|c|c|c|}
\hline № & Instrumento & Resultados \\
\hline 01 & $\begin{array}{l}\text { Escala Cornell de Depressão em } \\
\text { Demência - versão modificada }\end{array}$ & $\begin{array}{l}\text { Depressão prediz significativamente o funcionamento comportamental no âmbito da agressão física/abuso } \\
\text { verbal, intromissão e comportamento psicótico. }\end{array}$ \\
\hline 02 & $\begin{array}{l}\text { Escala de Depressão Geriátrica } \\
\text { - versão holandesa }\end{array}$ & $\begin{array}{l}\text { Depressão significativamente associada a: deficiência auditiva, ausência de filiação religiosa, incontinência, } \\
\text { internação hospitalar recente, cegueira, vulnerabilidade familiar, menos idade, solidão, dor, incapacidade } \\
\text { funcional, neuroticismo e falta de apoio social. }\end{array}$ \\
\hline 03 & $\begin{array}{l}\text { Escala de Depressão Geriátrica } \\
\text { de } 15 \text { itens - versão chinesa }\end{array}$ & $\begin{array}{l}\text { Depressão significativamente associada aos seguintes fatores socioeconômicos: não ser coberto pela } \\
\text { Assistência de Segurança Social, baixo nível de escolaridade e baixo nível de atividade social; e aos fatores } \\
\text { físicos: déficit visual, dificuldade de deglutição, baixa funcionalidade e à deambulação. Idosos depressivos } \\
\text { mais propensos à autopercepção de inadequação financeira, insatisfação com a vida, autopercepção de } \\
\text { saúde ruim, atitudes negativas em relação ao seu modo de vida e a relatar pensamentos suicidas recentes. }\end{array}$ \\
\hline 04 & $\begin{array}{l}\text { Escala de Depressão Geriátrica } \\
\text { de } 15 \text { itens - versão chinesa }\end{array}$ & $\begin{array}{l}\text { Em Taiwan, a depressão foi associada ao sexo feminino e baixa escolaridade e negativamente associada } \\
\text { à satisfação com a vida, condição de saúde autopercebida, percepção de adequação de renda, estado } \\
\text { funcional, estado cognitivo e satisfação com o apoio social. Sendo o gênero, a condição de saúde e a } \\
\text { adequação de renda preditores significativos da depressão. Em Honk Kong, a depressão foi significativa e } \\
\text { negativamente associada à satisfação com a vida, ao estado cognitivo, ao estado funcional (preditores), à } \\
\text { condição de saúde autopercebida, à percepção de adequação de renda e à rede de apoio social. }\end{array}$ \\
\hline 05 & Escala de Depressão Geriátrica & $\begin{array}{l}\text { Após seis meses de seguimento, o grupo deprimido apresentou mudanças significativamente mais graves } \\
\text { nos testes do Mini Exame do Estado Mental e Clinical Dementia Rating. }\end{array}$ \\
\hline 06 & Escala de Depressão Geriátrica & $\begin{array}{l}\text { Idosos institucionalizados sem atividade apresentaram maior tendência estatística para dar respostas } \\
\text { depressivas ao item } 2 \text { da GDS ("Você deixou muitos de seus interesses e atividades?"). GDS inversamente } \\
\text { correlacionada com o resultado do teste de fluência verbal. }\end{array}$ \\
\hline 07 & $\begin{array}{l}\text { Escala de Depressão Geriátrica } \\
\text { de } 15 \text { itens }\end{array}$ & $\begin{array}{l}\text { Depressão associada a maior idade, menor nível de escolaridade, pior estado funcional, menor satisfação } \\
\text { com a vida, pior estado de saúde percebido, diagnóstico de Hipertensão e Acidente Vascular Cerebral. } \\
\text { Estado depressivo consideravelmente prognosticado pelo pior estado funcional, insatisfação com a vida, } \\
\text { pior estado de saúde percebido e menor nível de escolaridade. }\end{array}$ \\
\hline
\end{tabular}


Quadro 2. (cont.)

08 Escala de Depressão Geriátrica

No início do estudo, a depressão foi associada à idade inferior a 80 anos, dor, deficiência visual, AVC, solidão e percepção de cuidados inadequados. Após seis meses sintomas depressivos foram significativamente associados à presença de sintomas depressivos na linha de base. Dor e limitações funcionais foram associadas com a presença de sintomas depressivos em seis meses. Maior nível de escolaridade associado à persistência de sintomas depressivos.

09 Escala Cornell de Depressão em Demência

10 Escala de depressão Geriátrica de 15 itens, Escala Cornell de Depressão em Demência, Montgomery and Asberg Depression Rating Scale, 17-item Hamilton Depression Rating Scale

11 Automated Geriatric Examination for Computer-assisted Taxonomy System (AGECAT)

12 Inventário de Depressão Geriátrica de Beck

13 Escala de Depressão Geriátrica de 15 itens

14 Taiwan Geriatric Depression Scale (TGDS)

15 Escala de Depressão Geriátrica de 15 itens

16 Escala de Depressão Geriátrica de 15 itens

17 InterRAI Long-Term Care Facilities Assessment System (InterRAI LTCF)

18 Minimum Data Set

19 Escala de Depressão Geriátrica de 10 itens

20 Escala de Depressão Geriátrica
Menores médias globais na escala de depressão associadas a maior poder de influência nas decisões políticas da instituição.

Depressão identificada pela GDS-15 associada ao luto pela perda de oportunidades e habilidades para participar de atividades, ao relato depressão prévia por um parente antes da admissão na instituição, não ajudar aos outros, não participar de atividades, dificuldade em resolver problemas e no estabelecimento de bons relacionamentos. GDS-15: correlação negativa fraca com idade para ambos os sexos. CSDD: correlação negativa fraca com idade para mulheres.

Depressão associada a menor idade e incapacidade funcional.

Baixa correlação negativa entre depressão e deterioração cognitiva.

Depressão associada de forma independente a ter filhos, maior idade, limitação funcional pela dor, pior estado cognitivo, menor mobilidade e menor pontuação no Índice de Barthel.

Depressão negativamente correlacionada com função cognitiva.

Para os homens, a depressão correlacionou-se negativamente com pontuações da MAN, hematócrito e hemoglobina. Para toda a população, correlacionou-se negativamente com pontuações da MAN.

Correlação negativa fraca entre as pontuações da GDS-15 e da MAN, mas as pontuações da MAN não foram significativamente maiores em idosos deprimidos. Depressão como único fator de risco independente para desnutrição.

A depressão foi mais frequentemente diagnosticada entre os idosos demenciados engajados socialmente. Dentre os idosos com alteração do humor, os demenciados eram menos susceptíveis a ser diagnosticados com transtorno depressivo em relação aos idosos não demenciados.

Institucionalização prévia fortemente associada com depressão na admissão. Uso prévio de antidepressivos fortemente associado com incidência de depressão após admissão. Depressão na admissão e sua incidência posterior foi significativamente menor nos idosos com comprometimento cognitivo. Maior uso de drogas psicotrópicas foi substancialmente associado a uma maior taxa de diagnósticos de depressão na admissão e nos trimestres posteriores. Dor e comorbidade física foram positivamente associadas com depressão identificada ao longo do primeiro ano.

Depressão significativamente mais elevada entre os moradores com quatro ou mais doenças crônicas, com polifarmácia, fazendo uso de medicamentos ansiolíticos e antipsicóticos, com aumento da dependência funcional, e autoavaliação e avaliação médica de saúde ruins.

Depressão associada a apego e integração social.

Fonte: Elaboração própria

\section{A Escala de Depressão Geriátrica (EDG)} foi o instrumento mais utilizado nos estudos analisados, tanto na versão de 30 itens (5 artigos), como de 15 itens (7 artigos) e 10 itens (1 artigo). Desenvolvida por Yesavage et al. (1983), é um dos instrumentos mais frequentemente aplicados por incluir uma pequena variação das respostas ( $\operatorname{sim} /$ não), não necessitar de um profissional da área da saúde mental para sua aplicação e poder ser autoaplicada ou aplicada por um entrevistador treinado. Sua versão original com 
30 itens vem cedendo espaço para a versão curta de 15 itens por reduzir significativamente o tempo gasto na aplicação e por ser confiável e válida entre idosos em diversas situações, sendo também recomendada para populações institucionalizadas (JONGENELIS ET AL., 2005).

A Escala Cornell de Depressão em Demência, segundo instrumento mais utilizado (3 artigos), foi desenvolvida por Alexopoulos et al. em 1988 e avalia alterações de humor, comportamento e distúrbios físicos e de ideação em pessoas com prejuízo cognitivo a partir de 19 itens aplicados pelo entrevistador ao paciente e ao cuidador, podendo também ser utilizada em indivíduos sem demência (PARADELA, 2011).

Observou-se também que em dois estudos (17 e 18) foram utilizados instrumentos que não são específicos para a avaliação da depressão, o Minimum Data Set e o InterRAI Long-Term Care Facilities Assessment System, os quais são formulários de coleta de dados que fornecem um panorama geral acerca das condições de saúde dos indivíduos residentes em instalações de longo prazo (BALLER ET AL., 2010; HOOVER ET AL., 2010).

Quanto ao objetivo desta revisão, percebeu-se que a maioria dos estudos testou a associação de sintomas depressivos com diversos tipos de variáveis, explorando uma ampla gama de aspectos relacionados à institucionalização. Ressalta-se a impossibilidade de estabelecer uma sequência temporal entre as associações encontradas, uma vez que a maioria das pesquisas apresentou delineamento transversal. A análise dos artigos permitiu a seleção de 6 fatores principais associados à depressão:

Fator 1: aspectos sociodemográficos. Os fatores sociodemográficos que apresentaram algum tipo de associação foram a idade (6 estudos), a escolaridade (4 estudos), a autopercepção de situação financeira ruim (2 estudos) e o sexo feminino (1 estudo). Houve divergências quanto à associação com a idade e escolaridade. No entanto, na maioria dos estudos, os idosos depressivos tinham menos idade $(02,08,10,11,13)$ e baixo nível de escolaridade $(03,04,07)$. Os eventos estressores da vida, possivelmente, são mais esperados e, por isso, melhor tolerados por idosos mais velhos, podendo, dessa maneira, não mais evocar depressão nesses indivíduos (EISSES ET AL., 2004). Além disso, os idosos com menos idade que vivem em Ilpi geralmente são os que apresentam mais doenças graves ou comprometimento cognitivo severo e, por isso, podem ser mais propensos a desenvolver sintomatologia depressiva (MCDOUGALL ET AL., 2007). O grau de instrução, associado à depressão, pode ser justificado por uma reação em cadeia, em que o baixo nível de escolaridade pode levar à inadequação de renda e impedir o acesso a cuidados de saúde, lazer e apoio social (KU; LIU; TSAI, 2006).

Fator 2: condições de saúde. A depressão foi mais frequentemente associada à autopercepção de saúde ruim $(03,04,07,19)$, à dor $(02,08,18)$, à deficiência visual $(02,03$, $08)$, à presença de comorbidades $(18,19)$, ao Acidente Vascular Cerebral (AVC) $(07,08)$ e ao maior risco de desnutrição $(15,16)$.

A autoavaliação da saúde é uma variável complexa que capta múltiplas dimensões da relação entre saúde física e outras características pessoais e sociais (DAMIÁN; BARRIUSO; VALDERRAMA-GAMA, 2008). No idoso, a autopercepção de saúde é influenciada pelas condições de suporte familiar, estado conjugal, oportunidades de educação e emprego, renda, capacidade funcional, condições crônicas de saúde, estilo de vida, dentre outros (HARTMANN, 2008). O processo de institucionalização favorece a vivência de perdas em vários desses aspectos da vida, aumentando a vulnerabilidade a quadros depressivos e contribuindo para uma pior percepção do estado de saúde (CARREIRA ET AL., 2011).

A dor é uma experiência vital que afeta de maneira integral o ser, ocasionando sofrimento, que quando prolongado e severo, como na maioria dos casos de dor crônica, afeta a qualidade de vida de quem a sofre de 
maneira significativa, gerando maior risco de comorbidade psiquiátrica, podendo esta relação ser de dupla via, sendo a dor fator de risco ou consequência de transtornos psíquicos (SERRANO, 2011). Em pessoas idosas, os mecanismos de cronificação da dor estão relacionados à diminuição da capacidade de adaptação que acompanha o envelhecimento, ao aumento da sensibilidade dolorosa, às comorbidades médicas e psiquiátricas e ao isolamento social (KARP ET AL., 2008).

Idosos com déficits visuais estão mais propensos a desenvolver quadros depressivos, uma vez que podem precisar alterar seu estilo de vida e sua independência funcional, requerendo mais assistência nas atividades básicas de vida diária, quando comparados com aqueles que não têm tal deficiência (MACEDO ET AL., 2008).

A associação entre doenças crônicas e sintomas depressivos é bimodal, de modo que a depressão pode precipitar o surgimento de doenças crônicas ou estas podem exacerbar sintomas depressivos por meio dos efeitos diretos na função cerebral ou através de alterações psicológicas e psicossociais (DUARTE; REGO, 2007).

A depressão está diretamente relacionada ao estado nutricional, uma vez que interfere no centro de controle neural, responsável pela fome, ansiedade e compulsões alimentares, podendo levar à desnutrição ou à obesidade (PEIXOTO, 2006). As questões econômicas, sociais, biológicas, psíquicas e alimentares também são determinantes do estado nutricional dos indivíduos idosos institucionalizados, tendo a depressão sido identificada como a maior causa de perda de peso nesse grupo populacional (VALENÇA; ANDRADE, 2011).

A relação entre depressão e AVC é complexa e também pode ocorrer nos dois sentidos. O surgimento de quadro depressivo em pacientes pós-AVC tende a ser visto como uma reação psicológica compreensível resultante da perda ou das incapacidades associadas à doença, sendo, por este motivo, subdiagnosticado (TERRONI, 2009). No primeiro ano após o AVC, considerado o período agudo, a depressão é a complicação psiquiátrica mais prevalente e a que tem sido mais associada a um pior prognóstico, uma vez que os pacientes com essa complicação apresentam recuperação funcional mais lenta, comprometimento das atividades da vida diária, internação hospitalar mais longa na fase aguda e maior mortalidade (SOUZA; TORQUATO JUNIOR; SOARES, 2010).

Fator 3: capacidade funcional. Baixa capacidade ou incapacidade funcional $(02,03$, 04, 07, 11, 13, 19) e limitação funcional pela dor (12) foram significativamente associados à sintomatologia depressiva nos idosos institucionalizados. Define-se como capacidade funcional a condição que o indivíduo possui de viver de maneira autônoma e de se relacionar em seu meio (NOGUeIRA ET AL., 2010). Pessoas idosas com depressão tendem a apresentar maior comprometimento físico, social e funcional, afetando sua qualidade de vida, cursando em redução ou perda da independência funcional (SANTOS ET AL., 2012).

Fator 4: comportamento. Vários tipos de comportamento foram associados à depressão, desde agressão física, abuso verbal e agir psicótico (01), neuroticismo (02), pensamentos suicidas recentes (03), indisponibilidade em ajudar aos outros e em participar de atividades, dificuldade em resolver problemas e em estabelecer bons relacionamentos (10). Dentre esses fatores, a ideação suicida, tentativas de suicídio e o suicídio merecem especial atenção, uma vez que representam instâncias da vida - expectativas, doença, sofrimento - que se tornaram difíceis de suportar, representando o auge da insatisfação com o viver (GREK, 2007). Investigações a respeito da ideação suicida são problemáticas, uma vez que esse fenômeno é pouco perguntado por pesquisadores e também muito pouco relatado pelos idosos, e, quando isso ocorre, pode ser confundido com processos depressivos (MINAYO; CAVALCANTE, 2010). A população institucionalizada tende a declarar com mais frequência a presença de pensamentos 
suicidas do que a não institucionalizada (ALMEIDA; QUINTÃO, 2012).

Fator 5: cognição. Os estudos também apresentaram divergências quanto a este aspecto. Em quatro deles, a depressão foi associada a um pior estado cognitivo $(04,05,13,14)$ e em duas pesquisas a um melhor estado cognitivo $(12,18)$. A grande frequência de queixas cognitivas em idosos depressivos levou à criação do termo pseudodemência depressiva, caso em que os sintomas de depressão são acompanhados pelas dificuldades de concentração e atenção e pela falta de memória (BANHATO, 2011). Muitas alterações cognitivas observadas em idosos com depressão se assemelham às observadas em quadros demenciais, enquanto outras se assemelham ao envelhecimento normal (ÁVILLA; BOTINO, 2006).

Fator 6: medicamentos. A polifarmácia (19) e o uso de antidepressivos e de psicotrópicos (18) foram associados à sintomatologia depressiva. Os pacientes internados em Ilpi merecem atenção especial quanto ao consumo de psicofármacos, tendo em vista seu uso corriqueiro em quadros demenciais, depressões e distúrbios comportamentais (LUCCHETTI ET AL., 2010). Estima-se que a prevalência de psicofármacos em asilados chegue a $63 \%$, sendo esses medicamentos usualmente prescritos por médicos não psiquiatras em decorrência da necessidade de controle comportamental, presença de sintomas de depressão e transtornos do sono (STELLA ET AL., 2006).

Outros fatores associados encontrados nos estudos incluídos na revisão que merecem destaque foram: a solidão $(02,08)$, depressão prévia $(08,10)$ e a falta de apoio social (02) ou insatisfação com o mesmo (04). Na velhice, o fenômeno depressivo em si pode representar a recorrência de episódios anteriores que se manifestaram em outras épocas da vida: pode ser a continuidade de uma depressão crônica anterior do tipo Distimia, pode ser uma depressão reativa, pode ainda surgir como consequência do prejuízo na qualidade de vida proporcionada por alguma outra doença orgânica concomitante ou, simplesmente, pode ser um episódio originado após os 60 anos (MARINHO, 2010).

Vivenciar a solidão pode indicar uma rede social insatisfatória e tem sido um dos fatores de risco para a depressão mais citado pelos diferentes investigadores, além de um dos principais motivos para a admissão em instituições (VAZ; GASPAR, 2011). Os eventos estressores, como o luto, situações em que há dificuldade de se estabelecer relações interpessoais e a falta de apoio social e familiar, também podem contribuir para a manifestação de sintomas depressivos (WHO, 2012).

\section{Considerações finais}

A presente revisão integrativa possibilitou caracterizar a produção científica acerca dos fatores significativamente associados à depressão em idosos institucionalizados. Apesar do considerável número de artigos incluídos, percebe-se uma lacuna com relação à categorização dos estudos devido à escassez de pesquisas de intervenção que retratassem evidências fortes, pois a maioria dos estudos encontrados tem delineamento transversal, classificado como nível VI de evidência, o que é considerado fraco.

Detectou-se, também, a baixa produção científica tanto no Brasil quanto na América Latina, sendo apenas dois artigos originários de pesquisas realizadas no Brasil, fazendo-se necessária uma maior reflexão sobre a relevância da temática no País, para que novos estudos sejam desenvolvidos e divulgados.

Durante a pré-seleção dos artigos, observou-se que muitos deles foram excluídos da revisão por não utilizarem técnicas estatísticas para testar hipóteses de associação da depressão com os fatores investigados. Os métodos estatísticos devem ser valorizados uma vez que aumentam a credibilidade das pesquisas e servem de base para as tomadas de decisões por parte dos gestores. 
Além disso, outros estudos, apesar de terem sido realizados em Ilpi, incluíram pessoas não idosas na amostra, descaracterizando o perfil do público-alvo desses locais.

Quanto aos instrumentos utilizados na detecção da depressão, observou-se que, apesar da ampla disponibilidade de instrumentos destinados a este fim na psiquiatria geriátrica, dois estudos utilizaram instrumentos de avaliação geral do estado de saúde, o que de certa maneira minimiza o poder de comparação dos resultados com aqueles estudos que utilizaram instrumentos específicos.

A variação dos fatores associados à depressão justifica-se pelas diferentes características das populações de cada país, pelos

\section{Referências}

ALEXOPOUlOS, G. S. Cornell Scale for Depression in dementia. Biological Psychiatry, San Francisco, v. 23, p. 271-84, 1988.

ALMEIDA, L.; QUINTÃO, S. Depressão e ideação suicida em idosos institucionalizados e não institucionalizados em Portugal. Acta Medica Portuguesa, Lisboa, v. 25, n. 6, p. 350-8, 2012. Disponível em: <http://www. actamedicaportuguesa.com/revista/index.php/amp/ article/view/1351/944>. Acesso em: 18 jan. 2013.

ÁVILA, R.; BOTTINO, C. M. C. Atualização sobre alterações cognitivas em idosos com síndrome depressiva. Revista Brasileira de Psiquiatria, São Paulo, v. 28, n. 4, p. 316-20, 2006. Disponível em: <http://www.scielo.br/ pdf/rbp/v28n4/2225.pdf>. Acesso em: 18 jan. 2013.

BALLER, M. Depression in Dutch homes for the elderly: under-diagnosis in demented residents? International Journal of Geriatric Psychiatry, Chichester, v. 25, n. 7, p. 712-8, 2010.

BANHATO, E. F. C. Validade de critério de uma forma abreviada da escala Wais-III em uma amostra de idosos brasileiros. 2011. $211 \mathrm{f}$. Tese (Doutorado em Saúde Brasileira) - Universidade Federal de Juiz de Fora, Juiz de Fora, 2011. objetivos específicos de cada pesquisa, pelos instrumentos utilizados e pelas técnicas de amostragem, o que resultou em algumas divergências de resultados para fatores semelhantes.

Essa observação é importante, uma vez que o intuito dessa revisão foi condensar as evidências científicas a respeito da temática em questão, cabendo aos profissionais da saúde que lidam com pessoas idosas institucionalizadas utilizar-se destes resultados no aprimoramento de suas práticas, valorizando as formas de investigação da depressão e dos possíveis fatores associados, considerando as particularidades e o contexto biopsicossocial e cultural de cada indivíduo.
BATISTA, A. S. Envelhecimento e dependência: desafios para a organização da proteção social. Brasília, DF: MPS: SPPS, 2008. (Coleção Previdência Social, v. 28).

BRASIL. Informe nacional sobre a implementação na América Latina e Caribe da Declaração de Brasília sobre Envelhecimento. 2012. Disponível em: <http://www. cepal.org/celade/noticias/paginas/9/46849/Brasil. pdf>. Acesso em: 21 dez. 2012.

Resolução da Diretoria Colegiada - $R D C n^{\circ} 283$, de 26 de setembro de 2005. Regulamento técnico para o funcionamento das Instituições de Longa Permanência para Idosos. Disponível em: <http://www.suvisa.rn.gov. br/contentproducao/aplicacao/sesap_suvisa/arquivos/ gerados/resol_rdc_283_set_2005.pdf >. Acesso em: 8 nov. 2012 .

CARREIRA, L. Prevalência de depressão em idosos institucionalizados. Revista Enfermagem UERJ, Rio de Janeiro, v. 19, n. 2, p. 268-273, 2011. Disponível em: <http://www.facenf.uerj.br/v19n2/v19n2al6.pdf>. Acesso em: 22 dez. 2012.

CRUZ, D. T.; CAETANO, V. C.; LEITE, I. C. G.

Envelhecimento populacional e bases legais da atenção à saúde do idoso. Cadernos de Saúde Coletiva, Rio 
de Janeiro, v. 18, n. 4, p. 500-508, 2010. Disponível em: $<$ http://www.iesc.ufrj.br/caderno/images/csc/2010_4/artigos/CSC_v18n4_500-508.pdf>. Acesso em: 28 nov. 2012.

DAMIÁN, J.; BARRIUSO, R. P.; VALDERRAMAGAMA, E. Factors associated with self-rated health in older people living in Institutions. BioMed Central Geriatrics, London, v. 8, n. 5, p. 1-6, 2008. Disponível em: <http://www.biomedcentral.com/content/ pdf/1471-2318-8-5.pdf >. Acesso em: 21 jan. 2013.

DUARTE, M. B.; REGO, M. A. V. Comorbidade entre depressão e doenças clínicas em um ambulatório de geriatria. Cadernos de Saúde Pública, Rio de Janeiro, v. 23, n. 3, p. 691-700, 2007. Disponível em: <http://www.scielo.br/ pdf/csp/v23n3/27.pdf>. Acesso em: 15 jan. 2013.

EISSES, A. M. H. Risk indicators of depression in residential homes. International Journal of Geriatric Psychiatry, Chichester, n. 19, v. 7, p. 634-40, 2004.

FARIA, F. A. C. Escolas promotoras de saúde na América Latina: uma revisão integrativa da literatura. 2010. 102 p. Dissertação (Mestrado em Promoção da Saúde) Universidade de Franca, Franca, 2010.

FERNANDES, M. G. M.; NASCIMENTO, N. F. S.; COSTA, K. N. F. M. Prevalência e determinantes de sintomas depressivos em idosos atendidos na atenção primária de saúde. Revista da Rede de Enfermagem do Nordeste, Fortaleza, v. 11, n. 1, p. 19-27, 2010. Disponível em: <http://www.revistarene.ufc.br/vollln1_html_site/ a02vllnl.htm>. Acesso em: 22 dez. 2012.

GALHARDO, V. A. C.; MARIOSA, M. A. S.; TAKATA, J. P. I. Depressão e perfis sociodemográfico e clínico de idosos institucionalizados sem déficit cognitivo. Revista Médica de Minas Gerais, Belo Horizonte, v. 20, n. 1, p. 16-21, 2010. Disponível em: <http://www.observatorionacionaldoidoso.fiocruz.br/biblioteca/_artigos/195.pdf>. Acesso em: 21 dez. 2012.

GIAVONI, A. Elaboração e validação da Escala de Depressão para Idosos. Cadernos de Saúde Pública, Rio de Janeiro, v. 24 n. 5, p. 975-982, 2008. Disponível em: $<$ http://www.scielo.br/pdf/csp/v24n5/04.pdf >. Acesso em: 22 dez. 2012.
GONZÁLEZ, L. A. M. Vivencia de los cuidadores familiares de adultos mayores que sufren depresión. Revista da Escola de Enfermagem da USP, São Paulo, v. 44, n. 1, p. 32-39, 2010. Disponível em: <http://www.observatorionacionaldoidoso.fiocruz.br/biblioteca/_artigos/166. pdf $>$. Acesso em: 28 jul. 2012.

GREK, A. Clinical management of suicidality in the elderly: an opportunity for involvement in the lives of older patients. Canadian Journal of Psychiatry, Ottawa, v. 52, n. 6 , supl. 1, p. 47S-57S, 2007.

HARTMANN JUNIOR, A. P.; SILVA, R. A.; BASTOS, O. Idosos institucionalizados: relação de estados depressivos com sintomas físicos e cognitivos. Neurobiologia, Recife, v. 72, n. 3, p. 19-30, 2009. Disponível em: <http:// www.neurobiologia.org/ex_2009.3/Revista\%20 Neurobilogia-72(3)2009\%20(Cap.3)(OK).pdf>. Acesso em: 21 dez. 2012.

HARTMANN, A. C. V. C. Fatores associados a autopercepção de saúde em idosos de Porto Alegre. 2008. 73 f. Tese (Doutorado em Gerontologia Biomédica) Pontifícia Universidade Católica do Rio Grande do Sul, Porto Alegre, 2008.

HOOVER, D. R. Depression in the first year of stay for elderly long-term nursing home residents in the U.S.A. International Psychogeriatrics, Cambridge, v. 22, n. 7, p. 1161-1171.

\section{INSTITUTO BRASILEIRO DE GEOGRAFIA E} ESTATÍSTICA (IBGE). Pesquisa Nacional por Amostra de Domicílios. 2012. Disponível em: <http://www.ibge. gov.br/home/presidencia/noticias/imprensa/ppts/00 000010135709212012572220530659 .pdf $>$. Acesso em: 18 dez. 2012.

JONGENELIS, K. Diagnostic accuracy of the original 30-item and shortened versions of the Geriatric Depression Scale in nursing home patients. International Journal of Geriatric Psychiatry, Chichester, v. 20, n. 11, p. 1067-1074, 2005.

KARP, J. Advances in understanding the mechanisms and management of persistent pain in older adults. British Journal of Anaesthesia, Oxford, v. 101, n. 1, p. 
111-120, 2008. Disponível em: <http://bja.oxfordjournals.org/content/101/1/111.full.pdf+html>. Acesso em: 22 jan. 2013.

KU, Y.; LIU, W.; TSAI, Y. Prevalence and risk factors for depressive symptoms among veterans home elders in Eastern Taiwan. International Journal of Geriatric Psychiatry, Chichester, v. 21, n. 12, p. 1181-8, 2006.

LONDON. Milton Keynes Primary Care Trust. Critical Appraisal Skills Programme. London: Oxford; 2002.

LUCCHETTI, G. Fatores associados ao uso de psicofármacos em idosos asilados. Revista de Psiquiatria do Rio Grande do Sul, Porto Alegre, v. 32, n. 2, p. 38-43, 2010. Disponível em: <http://www.scielo.br/pdf/rprs/ v32n2/v32n2a03.pdf >. Acesso em: 21 jan. 2013.

MACEDO, B. G. Impacto das alterações visuais nas quedas, desempenho funcional, controle postural e no equilíbrio dos idosos: uma revisão de literatura. Revista Brasileira de Geriatria e Gerontologia, Rio de Janeiro, v. 11, n. 3, p. 419-432, 2008. Disponível em: <http:// www.crde-unati.uerj.br/img_tse/v1ln3/capitulo9.pdf > . Acesso em: 20 jan. 2013.

MARINHO, S. M. B. A. O suporte social e a depressão no idoso. 2010. 102 f. Dissertação (Mestrado em Psicologia Clínica e da Saúde) - Universidade Fernando Pessoa, Porto, 2010.

MCDOUGALL, F. A. Prevalence and symptomatology of depression in older people living in institutions in England and Wales. Age and Ageing, Oxford, v. 36, n. 5, p. 562-568, 2007.

MEDEIROS, J. M. L. Depressão no idoso. 2010. 31 f. Dissertação (Mestrado Integrado em Medicina) Faculdade de Medicina, Universidade do Porto, Porto, 2010. Disponível em: <http://repositorio-aberto.up.pt/ bitstream/10216/53479/2/Depresso\%20no\%20Idoso. pdf >. Acesso em: 22 dez. 2012.

MELNYK, B. M. Evidence-based practice: step by step. The American Journal of Nursing, New York, v. 110, n. 5, p. 41-47, 2010.
MINAYO, M. C. S.; CAVALCANTE, F. G. Suicídio entre pessoas idosas: revisão da literatura. Revista de Saúde Pública [online], v. 44, n. 4, p. 750-757, 2010. Disponível em: <http://www.scielo.br/pdf/rsp/v44n4/20.pdf $>$. Acesso em: 15 jan. 2013.

NOGUEIRA, S. L. Fatores determinantes da capacidade funcional em idosos longevos. Revista Brasileira de Fisioterapia [online], v. 14, n. 4, p. 322-329, 2010. Disponível em: <http://www.scielo.br/pdf/rbfis/ v14n4/aop019_10.pdf >. Acesso em: 15 jan. 2013.

OLIVEIRA, J. L. M. Exercícios de resistência para a promoção de saúde de grupos especiais de coronariopatas. 2008. 62 p. Dissertação (Mestrado em Promoção da Saúde) - Universidade de Franca, Franca, 2008. Disponível em: $<$ http://www.dominiopublico.gov.br/download/texto/cp096082.pdf >. Acesso em: 15 dez. 2012.

PARADELA, E. M. P. Depressão em idosos. Revista do Hospital Universitário Pedro Ernesto, Rio de Janeiro, v. 10, n. 2, p. 31-40, 2011.

PEIXOTO, H. G. E. Estado nutricional e seus fatores interferentes em pacientes com transtorno depressivos. 2006. 61 f. Dissertação (Mestrado em Nutrição Humana) - Universidade de Brasília, Brasília.

PÓVOA, T. R. Prevalência de depressão nos idosos institucionalizados na morada do idoso do Instituto de Gerontologia de Brasília. Brasília Médica, Brasília, DF, v. 46, n. 3, p. 241-46, 2009. Disponível em: <http://www. ambr.com.br/rb/arquivos/08_bsb_med_46(3)_2009_ prevalencia_depressao.pdf >. Acesso em: 21 dez. 2012.

SAMPAIO, L. S. Condições sociodemográficas e de saúde de idosos residentes em domicílio no município de Jequié - BA. Revista Brasileira de Geriatria e Gerontologia, Rio de Janeiro, v. 12, n. 2, p. 267-274, 2009. Disponível em: $<$ http://www.crde-unati.uerj.br/img tse/v12n2/pdf/art_9.pdf $>$. Acesso em: 28 dez. 2012.

SANTANA, A. J.; BARBOZA FILHO, J. C. Prevalência de sintomas depressivos em idosos institucionalizados na cidade de Salvador. Revista Baiana de Saúde Pública, Salvador, v. 31, n. 1, p. 134-146, 2007. 
SANTOS, J. G. Sintomas depressivos e prejuízo funcional de idosos de um Centro-dia Geriátrico. Jornal Brasileiro de Psiquiatria, Rio de Janeiro, v. 61, n. 2, p. 102-106, 2012. Disponível em: <http://www.scielo.br/ pdf/jbpsiq/v6ln2/08.pdf>. Acesso em: 16 jan. 2013.

SERRANO, N. L. M. Importancia de la evaluación de aspectos psicosomáticos en los pacientes con dolor crónico neuropático. Acta Neurologica Colombiana, Bogotá, v. 27 n. 2, supl. 2, p. 113-124, 2011.

SIQUEIRA, G. R. Análise da sintomatologia depressiva nos moradores do abrigo Cristo Redentor através da aplicação da Escala de Depressão Geriátrica (EDG). Ciência \&t Saúde Coletiva, Rio de Janeiro, v. 14, n. 1, p. 253-259, 2009. Disponível em: <http://www.scielo.br/ pdf/csc/v14nl/a31v14nl.pdf >. Acesso em: 21 dez. 2012.

\section{SOUZA, B. P. F.; TORQUATO JUNIOR, M. A. A.;}

SOARES, S. M. S. R. Prevenção de depressão pós-AVC. Revista de Psiquiatria Clínica, São Paulo, v. 37, n. 4, p. 182 , 2010. Disponível em: <http://www.hcnet.usp.br/ipq/revista/vol37/n4/pdf/194.pdf >. Acesso em: 23 jan. 2013.

SOUZA, M. T.; SILVA, M. D.; CARVALHO, R. Revisão integrativa: o que é e como fazer? Einstein, São Paulo, v. 8, n. 1, p. 102-106, 2010. Disponível em: <http://apps. einstein.br/revista/arquivos/PDF/1134-Einsteinv8n1_ p102-106_port.pdf $>$. Acesso em: 5 nov. 2012.

SOUZA, P. D. Aptidão funcional de idosos residentes em uma instituição de longa permanência. Revista Brasileira de Geriatria e Gerontologia, Rio de Janeiro, v. 14, n. 1, p. 7-16, 2011. Disponível em: <http://revista. unati.uerj.br/pdf/rbgg/v14nl/v14nla02.pdf > . Acesso em: 21 dez. 2012.

STELLA, F. Factors influencing psychotropic prescription by non-psychiatrist physicians in a nursing home for the elderly in Brazil. São Paulo Medical Journal, São Paulo, v. 124, n. 5, p. 253-6, 2006. Disponível em: <http://www.scielo.br/pdf/spmj/v124n5/03.pdf>. Acesso em: 21 jan. 2013.

TERRONI, L. M. N. Associação entre o episódio depressivo maior após acidente vascular cerebral isquêmico e comprometimento de circuitos neuronais pela lesão: um estudo prospectivo de 4 meses. 2009. 146 f. Tese (Doutorado em Ciências) - Faculdade de Medicina da Universidade de São Paulo, São Paulo, 2009.

TOLEDO, M. M. Vulnerabilidade do adolescente ao HIV/AIDS: revisão integrativa. 2008. 153f. Dissertação (Mestrado em Enfermagem em Saúde Coletiva) Escola de Enfermagem, Universidade de São Paulo, São Paulo, 2008

VALCARENGHI, R. V. Alterações na funcionalidade/ cognição e depressão em idosos institucionalizados que sofreram quedas. Acta Paulista de Enfermagem, São Paulo, v. 24 n. 6, p. 828-33, 2011. Disponível em: <http://www.scielo.br/pdf/ape/v24n6/a17v24n6.pdf>. Acesso em: 21 dez. 2012.

VALENÇA, J. M.; ANDRADE, K. L. Desnutrição associada à depressão em idosos hospitalizados. Geriatria $\mathcal{E}$ Gerontologia, Rio de Janeiro, v. 5, n. 1, p. 14-18, 2011.

VAZ, S. F. A.; GASPAR, N. M. S. Depressão em idosos institucionalizados no distrito de Bragança. Revista de Enfermagem Referência [online], v. 3, n. 4, p. 49-58, 2011. Disponível em: <http://www.scielo.gpeari.mctes. $\mathrm{pt} / \mathrm{pdf} / \mathrm{ref} /$ vserIIIn4/serIIIn4a05.pdf $>$. Acesso em: 30 jan. 2013

WORLD HEALTH ORGANIZATION (WHO) Conquering Depression. 2012. Disponível em: <http:// www.searo.who.int/en/Section1174/Section1199/ Section1567/Section1826.htm>. Acesso em: 11 jan. 2013.

YESAVAGE, J. A. Development and validation of a geriatric depression screening scale: a preliminary report. Journal of Psychiatric Research, Londres, v. 17, n. 1, p. 37-49, 1983. Disponível em: <http://home. uchicago.edu/ /tmurrayl/research/articles/at\%20 least\%20read/development\%20and\%20validation\%20 of $\% 20$ a\%20geriatric\%20depression $\% 20$ screening $\% 20$ scale_a\%20preliminary\%20report.pdf $>$. Acesso em: 5 dez. 2012.

\footnotetext{
Recebido para publicação em dezembro de 2013

Versão final em agosto de 2014

Conflito de interesses: inexistente
}

Suporte financeiro: não houve 